\title{
Distributed Coordination Control of First- and Second-Order Multiagent Systems with External Disturbances
}

\author{
Lipo Mo, ${ }^{1}$ Tingting Pan, ${ }^{1}$ Shaoyan Guo, ${ }^{1}$ and Yuguang Niu ${ }^{2}$ \\ ${ }^{1}$ School of Science, Beijing Technology and Business University, Beijing 100048, China \\ ${ }^{2}$ State Key Laboratory of Alternate Electrical Power System with Renewable Energy Sources, North China Electric Power University, \\ Beijing 102206, China \\ Correspondence should be addressed to Lipo Mo; molipo@th.btbu.edu.cn
}

Received 7 October 2015; Revised 25 October 2015; Accepted 27 October 2015

Academic Editor: Peng Lin

Copyright (C) 2015 Lipo Mo et al. This is an open access article distributed under the Creative Commons Attribution License, which permits unrestricted use, distribution, and reproduction in any medium, provided the original work is properly cited.

\begin{abstract}
This paper is devoted to the coordination control problem of heterogeneous first- and second-order multiagent systems with external disturbances. First, by applying the theory of eigenvalue and the method of model transformation, the consensus state of heterogeneous multiagent systems is obtained. Then, based on the consensus state, the control output is defined, and sufficient conditions are derived to make all agents reach consensus with $H_{\infty}$ performance. Finally, simulation results are provided to demonstrate the effectiveness of the presented results.
\end{abstract}

\section{Introduction}

In the past decade, the consensus problem of multiagent systems is a hot issue in the field of systems and control, partly due to their potential applications in practical systems, such as unmanned vehicles and automated highway systems. For multiagent systems with first-order, second-order, highorder, and fractional-order integrator, many protocols have been designed based on the information of each agent and its neighbors [1]. For example, Vicsek et al. proposed a simple model for phase transition of a group of self-driven particles and numerically demonstrated complex dynamics of the model [2], and then Jadbabaie et al. provided a theoretical explanation for the consensus behavior of the Vicsek model using graph theory [3]. Hong et al. studied the consensus problem of multiagent systems with switching jointly connected topologies [4]. The consensus problem for multiagent systems with time delays is investigated in [5-10]. And then the leader-following consensus protocols are given in $[11,12]$. Recently, the consensus problem for fractionalorder multiagent systems is also investigated, such as [13]. In [14-16], rotating and constrained consensus problems are considered, respectively, and some new protocols are proposed.
Most of results of consensus problem are on multiagent systems with the same-order dynamics. Recently, the consensus of heterogeneous multiagent systems has received more and more attention because the dynamics of the agents coupled with each other are different in the practical systems. The consensus problem of heterogeneous multiagent systems with linear consensus protocol and saturated consensus protocol was studied in [17]; some sufficient conditions for consensus are established when the communication topologies are undirected connected graphs. Then, the consensus problem of heterogeneous multiagent systems with directed communication graphs was investigated in [18]. And the consensus problem of heterogeneous multiagent systems composed of first-order, second-order, and nonlinear EulerLagrange agents was considered in [19]. In [20], the consensus problem for heterogeneous multiagent systems with switching jointly connected interconnection is studied. In engineering, it is necessary to know the consensus state of the system. However, most of results have not given the consensus state of heterogeneous multiagent systems. In the past decade, the consensus states are deduced for some protocols, however, most of which include the negative feedback of velocity for every second-order agent. But what is the consensus state when the protocol does not include the 
negative feedback of velocity? This paper will investigate this problem.

On the other hand, multiagent systems are often subjected to various disturbances such as actuator bias, measurement/calculation errors, and the variation of the communication topology. For homogeneous multiagent systems, robust $H_{\infty}$ consensus problems for first-order and high-order multiagent systems with external disturbances were studied in [21-23]. And some conditions are derived to make all agents reach consensus with $H_{\infty}$ performance. However, to the best of our knowledge, there is little result on the $H_{\infty}$ consensus problem of heterogeneous multiagent systems.

Compared with literatures, agents considered in this paper not only have different order integrator dynamics but also are influenced by external disturbances. First, we deduced the consensus values of heterogeneous multiagent systems by applying the theory of linear algebra and the method of model transformation. Based on the consensus state, the control output is defined, and then we gave the sufficient conditions under which all agents can reach consensus with $H_{\infty}$ performance by applying the robust control method. Finally, we demonstrated the effectiveness of the presented results by simulation.

\section{Preliminaries}

2.1. Graph Theory. To solve the coordination problems, graph theory is useful (see [24] for details). Consider a dynamical system consisting of $n$ agents. With regard to the $n$ agents as the vertices $V=\left\{v_{i}, i=1,2, \ldots, n\right\}$, the interconnection topology of $n$ agents can be conveniently described by an undirected graph $G=\{V, \varepsilon\}$, where $\varepsilon \subset V \times V$ is the set of edges of the graph. $\left(v_{i}, v_{j}\right)$ defines one of the graph's edges if $v_{i}$ and $v_{j}$ can communicate with each other. $N_{j}(t)=\{i \mid$ $\left.\left(v_{i}, v_{j}\right) \in \mathcal{E}\right\}$ denotes the set of labels of those agents which are neighbors of agent $i(i=1,2, \ldots, n)$ at time $t$. Let $A=$ $\left[a_{i j}\right] \in R^{n \times n}$ be the weighted adjacency matrix of the graph $G$, and $D=\operatorname{diag}\left\{d_{1}, d_{2}, \ldots, d_{n}\right\} \in R^{n \times n}$ is its degree matrix, where $d_{i}=\sum_{j=1}^{n} a_{i j}, i=1, \ldots, n$. Then the Laplacian of the weighted graph is defined as $L=D-A$, which is symmetric. A path that connects $v_{i}$ and $v_{j}$ in the graph $G$ is a sequence of distinct vertices $v_{i_{0}}, \ldots, v_{i_{m}}$, where $v_{i_{0}}=v_{i}, v_{i_{m}}=v_{j}$, and $\left(v_{i_{r}}, v_{i_{r+1}}\right) \in \varepsilon, 0 \leq r \leq m-1$. If there exists a path between any two vertices $v_{i}$ and $v_{j}(i \neq j)$, then the graph is said to be connected.

2.2. System Model. Consider a heterogeneous multiagent system composed of first-order and second-order integrator agents. The number of agents is $n$. Assume that there are $m$ second-order integrator agents $(m<n)$. And each secondorder agent dynamics is given as follows:

$$
\begin{aligned}
& \dot{x}_{i}(t)=v_{i}(t), \\
& \dot{v}_{i}(t)=u_{i}(t)+w_{i}(t), \quad i \in \mathscr{I}_{m},
\end{aligned}
$$

where $x_{i} \in \mathscr{R}, v_{i} \in \mathscr{R}$, and $u_{i} \in \mathscr{R}$ are the position, velocity, and control input of agent $i$, respectively, $\mathscr{I}_{m}=\{1,2, \ldots, m\}$, and $w_{i}(t) \in L_{2}$ is the external disturbance. Each first-order agent dynamics is given as follows:

$$
\dot{x}_{i}(t)=u_{i}(t)+w_{i}(t), \quad i \in \mathscr{I}_{n}-\mathscr{I}_{m},
$$

where $x_{i} \in \mathscr{R}$ and $u_{i} \in \mathscr{R}$ are the position and control input of agent $i$, respectively, $w_{i}(t) \in L_{2}$ is the external disturbance, and $\mathscr{I}_{n}=\{1,2, \ldots, n\}$. Let $x(0)=\left[x_{1}(0), \ldots, x_{n}(0)\right]^{T}$ and $v(0)=\left[v_{1}(0), \ldots, v_{m}(0)\right]^{T}$ be the initial condition of system ((1)-(2)).

Definition 1. The heterogeneous multiagent system ((1)-(2)) with $w_{i}=0(\forall i)$ is said to reach consensus if, for any initial condition, one has

$$
\begin{aligned}
& \lim _{t \rightarrow \infty}\left\|x_{i}(t)-x_{j}(t)\right\|=0, \quad \forall i, j \in \mathscr{I}_{n}, \\
& \lim _{t \rightarrow \infty}\left\|v_{i}(t)-v_{j}(t)\right\|=0, \quad \forall i, j \in \mathscr{I}_{m} .
\end{aligned}
$$

Partition the adjacency matrix $A$ of $G$ as $A=\left[\begin{array}{ll}A_{s} & A_{s f} \\ A_{f s} & A_{f}\end{array}\right]$, where $A_{s} \in \mathscr{R}^{m \times m}, A_{f} \in \mathscr{R}^{(n-m) \times(n-m)}, A_{s f} \epsilon$ $\mathscr{R}^{m \times(n-m)}$, and $A_{f s} \in \mathscr{R}^{(n-m) \times m}$. Let $L_{s}$ and $L_{f}$ be the Laplacian matrix of the second-order and first-order agents networks, respectively. For agent $i$, let $N_{i, s}$ be its neighbors with second-order agents and let $N_{i, f}$ be its neighbors with first-order agents. Thus, the Laplacian matrix of $G$ can be written as

$$
L=\left[\begin{array}{cc}
L_{s}+D_{s f} & -A_{s f} \\
-A_{f s} & L_{f}+D_{f_{s}}
\end{array}\right],
$$

where

$$
\begin{aligned}
& D_{s f}=\operatorname{diag}\left\{\sum_{j \in N_{1, f}} a_{1 j}, \ldots, \sum_{j \in N_{m, f}} A_{m j}\right\}, \\
& D_{f s}=\operatorname{diag}\left\{\sum_{j \in N_{m+1, s}} a_{m+1, j}, \ldots, \sum_{j \in N_{n, s}} A_{n j}\right\} .
\end{aligned}
$$

In this paper, we apply the following linear consensus protocol to the heterogeneous multiagent system ((1)-(2)):

$$
\begin{aligned}
& u_{i}(t) \\
& = \begin{cases}\sum_{j=1}^{n} a_{i j}\left(x_{j}-x_{i}\right)+k_{1} \sum_{j=1}^{m} a_{i j}\left(v_{j}-v_{i}\right), & i \in \mathscr{I}_{m}, \\
k_{2} \sum_{j=1}^{n} a_{i j}\left(x_{j}-x_{i}\right), & i \in \mathscr{I}_{n}-\mathscr{I}_{m},\end{cases}
\end{aligned}
$$

where $A=\left[a_{i j}\right]$ is the corresponding weighted adjacency matrix and $k_{1}$ and $k_{2}$ are the control parameters.

Remark 2. In [17], the convergence of system ((1)-(2)) with protocol (6) is proved when $w=0$, but the consensus state is not provided. In [18], the consensus states are deduced for some protocols; however, all protocols considered there 
include the absolute information of velocity for every agent. In fact, for practical systems, the absolute information of velocity can not be received sometimes. Therefore, it is meaningful to study the consensus state of system ((1)-(2)) with protocol (6).

Let $x_{s}(t)=\left[x_{1}(t), \ldots, x_{m}(t)\right]^{T}, v_{s}(t)=\left[v_{1}(t), \ldots\right.$, $\left.v_{m}(t)\right]^{T}, x_{f}(t)=\left[x_{m+1}(t), \ldots, x_{n}(t)\right]^{T}, y(t)=\left[x_{s}(t)^{T}, v_{s}(t)^{T}\right.$, $\left.x_{f}(t)^{T}\right]^{T}$, and $w(t)=\left[w_{1}, \ldots, w_{n}\right]^{T}$. Then, the dynamics of system ((1)-(2)) can be written as

$$
\dot{y}(t)=\Gamma y(t)+\Gamma^{\prime} w(t),
$$

where

$$
\begin{aligned}
\Gamma & =\left[\begin{array}{ccc}
0 & E_{m} & 0 \\
-L_{s}-D_{s f} & -k_{1} L_{s} & A_{s f} \\
k_{2} A_{f s} & 0 & -k_{2}\left(L_{f}+D_{f s}\right)
\end{array}\right], \\
\Gamma^{\prime} & =\left[\begin{array}{c}
0_{m \times n} \\
E_{n}
\end{array}\right],
\end{aligned}
$$

and $E_{n}$ is the identity matrix of order $n$. If $w(t)=0$, then system (7) can be changed into the following form:

$$
\dot{y}(t)=\Gamma y(t) .
$$

\section{Main Results}

In this section, firstly, the final consensus values of heterogeneous multiagent system (9) are deduced by applying matrix theory. The following lemmas are necessary in the following analysis.

Lemma 3 (see [24]). Let $G$ be a graph on $n$ vertices with Laplacian $L$. Denote the eigenvalues of $L$ by $\lambda_{1}(L), \ldots, \lambda_{n}(L)$ satisfying $\lambda_{1}(L) \leq \cdots \leq \lambda_{n}(L)$. Then $\lambda_{1}(L)=0$ and $\mathbf{1}=$ $[1,1, \ldots, 1]^{T} \in \mathscr{R}^{n}$ is the eigenvector associated with zero eigenvalue. Moreover, if $G$ is connected, then $\lambda_{2}(L)>0$.

Lemma 4 (see [17]). Suppose that the communication network $G$ is undirected and connected graph. Then the consensus of the heterogeneous multiagent system ((1)-(2)) with $w=0$ can be achieved by consensus protocol (6).

Lemma 5. Suppose that the communication network $G$ is an undirected and connected graph. Then the system matrix $\Gamma$ has a simple zero eigenvalue. In addition, all the other eigenvalues are less than zero.

Proof. It is easy to see that zero is the eigenvalue of $\Gamma$. By the elementary transformation of matrix, we have

$$
\Gamma \longrightarrow\left[\begin{array}{cc}
E_{m} & 0 \\
0 & L
\end{array}\right]
$$

where $L$ is the Laplacian matrix of $G$. From Lemma 3, we have $\operatorname{rank}(L)=n-1 ;$ thus, $\operatorname{rank}(\Gamma)=n+m-1$. So zero is the simple eigenvalue of $\Gamma$. On the other hand, system (9) is stable by Lemma 4, so the eigenvalues of $\Gamma$ have nonpositive real parts. Therefore, all the other eigenvalues of $\Gamma$ have negative real parts.

Next, we will give the main result of this section.

Theorem 6. Consider the heterogeneous multiagent system ((1)-(2)) with protocol (6). Suppose that the communication topology $G$ is undirected and connected. Then one has

$$
\lim _{t \rightarrow \infty} x_{i}(t)=\frac{k_{2}}{n-m} \sum_{i=1}^{m} v_{i}(0)+\frac{1}{n-m} \sum_{i=m+1}^{n} x_{i}(0),
$$

$$
i \in \mathscr{I}_{n}
$$

$\lim _{t \rightarrow \infty} v_{i}(t)=0, \quad i \in \mathscr{I}_{m}$,

when $w(t)=0$.

Proof. It is easy to see that $\alpha=\left[\mathbf{1}_{m}^{T}, 0, \mathbf{1}_{n-m}^{T}\right]^{T}$ is the right eigenvector of $\Gamma$ associated with zero eigenvalue and $\beta^{T}=$ $\left[0, \epsilon k_{2} \mathbf{1}_{m}^{T}, \epsilon \mathbf{1}_{n-m}^{T}\right]$ is the left eigenvector of $\Gamma$ associated with zero eigenvalue, where $\epsilon$ is a parameter to be determined.

From matrix theory and $\operatorname{rank}(\Gamma)=n+m-1$, we know that there exists a nonsingular matrix $P=\left[\begin{array}{ll}\alpha & P_{1}\end{array}\right]$ with $P^{-1}=$ $\left[\begin{array}{ll}\beta & P_{2}\end{array}\right]^{T}$, such that

$$
P^{-1} \Gamma P=J=\left[\begin{array}{ll}
0 & 0 \\
0 & J_{1}
\end{array}\right],
$$

where $P_{1}, P_{2} \in \mathscr{R}^{(n+m) \times(n+m-1)}, J$ is the Jordan canonical form of $\Gamma$, and $J_{1} \in \mathscr{R}^{(n+m-1) \times(n+m-1)}$. Since $P^{-1} P=E$, we have $\beta^{T} \alpha=1$; thus, $\epsilon=1 /(n-m)$. Therefore, $\beta=\left[0\left(k_{2} /(n-\right.\right.$ $\left.m)) \mathbf{1}_{m}^{T}(1 /(n-m)) \mathbf{1}_{n-m}^{T}\right]^{T}$.

On the other hand, noting that $\Gamma=P J P^{-1}$ and all the eigenvalues of $J_{1}$ have negative real parts, we have that $\lim _{t \rightarrow \infty} e^{\Gamma t}=\lim _{t \rightarrow \infty} P e^{J t} P^{-1}=\alpha \beta^{T}$. By the theory of linear systems, it is easy to see that

$$
\begin{aligned}
& \lim _{t \rightarrow \infty}\left[\begin{array}{c}
x_{s}(t) \\
v_{s}(t) \\
x_{f}(t)
\end{array}\right]=\lim _{t \rightarrow \infty} e^{\Gamma t}\left[\begin{array}{c}
x_{s}(0) \\
v_{s}(0) \\
x_{f}(0)
\end{array}\right] \\
& =\left[\begin{array}{c}
\frac{k_{2}}{n-m} \mathbf{1}_{m} \mathbf{1}_{m}^{T} v_{s}(0)+\frac{1}{n-m} \mathbf{1}_{m} \mathbf{1}_{n-m}^{T} x_{f}(0) \\
0 \\
\frac{k_{2}}{n-m} \mathbf{1}_{n-m} \mathbf{1}_{m}^{T} v_{s}(0)+\frac{1}{n-m} \mathbf{1}_{n-m} \mathbf{1}_{n-m}^{T} x_{f}(0)
\end{array}\right] .
\end{aligned}
$$

Therefore,

$$
\begin{aligned}
& \lim _{t \rightarrow \infty} x_{i}(t)=\frac{k_{2}}{n-m} \sum_{i=1}^{m} v_{i}(0)+\frac{1}{n-m} \sum_{i=m+1}^{n} x_{i}(0), \\
& \lim _{t \rightarrow \infty} v_{i}(t)=0, \quad i \in \mathscr{I}_{n} ;
\end{aligned}
$$


Remark 7. Theorem 6 implies that the consensus position of all agents depends only on velocities of second-order agents, positions of first-order agents, and feedback gain $k_{2}$ and has no relation of the positions of second-order agents and feedback gain $k_{1}$. The consensus velocities of agents are zero even though there is no negative feedback of velocities in protocol (6).

Remark 8. In [18], the consensus states of heterogeneous multiagent systems are deduced for some protocols which include the absolute information of velocity of every secondorder agent, and the analysis was complex. In the paper, we consider different protocol (6), which only includes relative information of agents, and the analysis is more comprehensive and obvious. And protocol (6) is not the special case of [18] because formula (10) in [18] can not be satisfied herein.

Next, we will investigate $H_{\infty}$ performance of system ((1)(2)) using consensus protocol (6). The following lemma is helpful in the consensus analysis.

Lemma 9 (see [25]). Consider the system

$$
\begin{aligned}
& \dot{x}(t)=A x(t)+B w(t), \\
& z(t)=C x(t),
\end{aligned}
$$

where $x(t) \in \mathscr{R}^{n}, w(t) \in \mathscr{R}^{l}$, and $z(t) \in \mathscr{R}^{p}$ are the state, input, and output of system (15). The systems matrices A, $B, C$ are constant matrices of appropriate dimensions. Given $\gamma>0$, system (15) is asymptotically stable with $\left\|T_{z w}(s)\right\|<\gamma$ for all nonzero $w(t) \in L_{2}[0, \infty)$, if there exists a positive definite matrix $Q \in \mathscr{R}^{n \times n}$, such that the following linear matrix inequality holds:

$$
\left[\begin{array}{ccc}
A^{T} Q+Q A & Q B & C^{T} \\
B^{T} Q & -\gamma E & 0 \\
C & 0 & -\gamma E
\end{array}\right]<0 .
$$

The objective of this paper is to find appropriate parameters of protocol (6) to attenuate disturbances of agents and make all agents reach consensus. Considering the consensus state of system (9), a natural way to define control output $z_{i}(t)$ is as follows:

$$
\begin{aligned}
& z_{i}(t)=x_{i}(t)-\frac{k_{1}}{n-m} \sum_{j=1}^{m} v_{j}(t)-\frac{1}{n-m} \sum_{j=m+1}^{n} x_{j}(t), \\
& z_{i}(t)=v_{i}(t), \quad i \in \mathcal{I}_{m}, \\
& z_{i}(t)=x_{i-m}-\frac{k_{1}}{n-m} \sum_{j=1}^{m} v_{j}(t)-\frac{1}{n-m} \sum_{j=m+1}^{n} x_{j}(t), \\
& i \in\{2 m+1, \ldots, m+n\} .
\end{aligned}
$$

Let $z(t)=\left[z_{1}(t), \ldots, z_{n+m}(t)\right]$; then (17) can be summarized as

$$
\begin{aligned}
z(t) & =y(t)-\frac{k_{2}}{n-m} \alpha \sum_{i=1}^{m} v_{i}(t)-\frac{1}{n-m} \alpha \sum_{i=m+1}^{n} x_{i}(t) \\
& =\left(E-\alpha \beta^{T}\right) y(t)=C y(t),
\end{aligned}
$$

where $C=E-\alpha \beta^{T}$.

Theorem 10. Consider system ((1)-(2)), (17) with control protocol (6). Given $\gamma>0$, suppose that the communication topology $G$ is undirected and connected. Then consensus can be achieved with $\left\|T_{z w}(s)\right\|_{\infty}<\gamma$ if there exists a positive definite matrix $Q \in \mathscr{R}^{(n+m-1) \times(n+m-1)}$ such that

$$
\left[\begin{array}{ccc}
J_{1}^{T} Q+Q J_{1} & Q \bar{\Gamma} & \bar{C} \\
\bar{\Gamma}^{T} Q & -\gamma E & 0 \\
\bar{C} & 0 & -\gamma E
\end{array}\right]<0,
$$

where $\bar{\Gamma}=P_{1} \Gamma^{\prime}, \bar{C}=\left(E-\alpha \beta^{T}\right) P_{1}$.

Proof. Let $\bar{y}(t)=y(t)-\left(k_{2} /(n-m)\right) \alpha \sum_{i=1}^{m} \int_{0}^{t} w_{i}(s) d s-$ $(1 /(n-m)) \alpha \sum_{i=m+1}^{n} \int_{0}^{t} w_{i}(s) d s=y(t)-\alpha \beta^{T} \kappa$, where $\alpha$ is defined as in the proof of Theorem 6 and $\kappa=$ $\left[0_{m}, \int_{0}^{t} w_{1}(s) d s, \ldots, \int_{0}^{t} w_{n}(s) d s\right]$. Define

$$
\left[\begin{array}{l}
\xi^{1}(t) \\
\xi(t)
\end{array}\right]=P^{-1} \bar{y}(t) .
$$

Noting that $\Gamma \alpha=0, P_{2}^{T} \alpha=0$, and $\beta^{T} \alpha=1$, we have

$$
\begin{aligned}
& {\left[\begin{array}{c}
\dot{\xi}^{1} \\
\dot{\xi}
\end{array}\right]=P^{-1} \frac{d}{d t} \bar{y}(t)=P^{-1}\left[\dot{y}(t)-\frac{k_{2}}{n-m} \alpha \sum_{i=1}^{m} w_{i}(t)\right.} \\
& \left.-\frac{1}{n-m} \alpha \sum_{i=m+1}^{n} w_{i}(t)\right]=P^{-1}\left[\Gamma y(t)+\Gamma^{\prime} w(t)\right. \\
& \left.-\frac{k_{2}}{n-m} \alpha \sum_{i=1}^{m} w_{i}(t)-\frac{1}{n-m} \alpha \sum_{i=m+1}^{n} w_{i}(t)\right] \\
& =P^{-1}\left[\Gamma_{\bar{y}}(t)+\Gamma^{\prime} w(t)-\frac{k_{2}}{n-m} \alpha \sum_{i=1}^{m} w_{i}(t)\right. \\
& \left.-\frac{1}{n-m} \alpha \sum_{i=m+1}^{n} w_{i}(t)\right]=P^{-1} \Gamma P\left[\begin{array}{l}
\xi^{1}(t) \\
\xi(t)
\end{array}\right] \\
& +P^{-1} \Gamma^{\prime} w(t)-\frac{k_{2}}{n-m} P^{-1} \alpha \sum_{i=1}^{m} w_{i}(t)-\frac{1}{n-m} \\
& \cdot P^{-1} \alpha \sum_{i=m+1}^{n} w_{i}(t)=\left[\begin{array}{ll}
0 & 0 \\
0 & J_{1}
\end{array}\right]\left[\begin{array}{l}
\xi^{1}(t) \\
\xi(t)
\end{array}\right]
\end{aligned}
$$




$$
\begin{aligned}
& +\left[\begin{array}{c}
\beta^{T} \\
P_{2}^{T}
\end{array}\right]\left[\begin{array}{c}
0_{m \times n} \\
E_{n}
\end{array}\right] w(t)-\frac{k_{2}}{n-m}\left[\begin{array}{c}
\beta^{T} \\
P_{2}^{T}
\end{array}\right] \alpha \sum_{i=1}^{m} w_{i}(t) \\
& -\frac{1}{n-m}\left[\begin{array}{c}
\beta^{T} \\
P_{2}^{T}
\end{array}\right] \alpha \sum_{i=m+1}^{n} w_{i}(t)=\left[\begin{array}{c}
0 \\
J_{1} \xi(t)
\end{array}\right] \\
& +\left[\begin{array}{c}
0 \\
P_{2}^{T} \Gamma^{\prime} w(t)
\end{array}\right]
\end{aligned}
$$

Thus, system (7) can be changed into

$$
\begin{aligned}
\dot{\xi}^{1}(t) & =0, \\
\dot{\xi}(t) & =J_{1} \xi(t)+P_{2} \Gamma^{\prime} w(t) .
\end{aligned}
$$

The first equation of (22) implies that $\left\{\dot{\xi}^{1}(t)=0\right\}$ is an invariant manifold, and the position variables converge to it. To investigate $H_{\infty}$ performance of heterogeneous multiagent systems, we need only to study the second equation of system (22). On the other hand, $z(t)=C y(t)=\left(E-\alpha \beta^{T}\right)(\bar{y}(t)+$ $\left.\alpha \beta^{T} \kappa\right)$. Noting that $\left(E-\alpha \beta^{T}\right) \alpha \beta^{T}=0$ and $\left(E-\alpha \beta^{T}\right) \alpha=0$, we have

$$
\begin{aligned}
z(t) & =\left(E-\alpha \beta^{T}\right) \bar{y}(t)=\left(E-\alpha \beta^{T}\right)\left[\begin{array}{ll}
\alpha & P_{1}
\end{array}\right]\left[\begin{array}{c}
\xi^{1}(t) \\
\xi(t)
\end{array}\right] \\
& =\left(E-\alpha \beta^{T}\right) P_{1} \xi(t)=\bar{C} \xi(t),
\end{aligned}
$$

where $\bar{C}=\left(E-\alpha \beta^{T}\right) P_{1}$.

Thus, $H_{\infty}$ consensus problem of system ((1), (2), (6)), (17) can be changed to $H_{\infty}$ consensus problem of the following system:

$$
\begin{aligned}
& \dot{\xi}(t)=J_{1} \xi(t)+P_{2}^{T} \Gamma^{\prime} w(t), \\
& z(t)=\bar{C} \xi(t) .
\end{aligned}
$$

By Lemma 9, system (24) is asymptotically stable with $\left\|T_{z w}(s)\right\|_{\infty}<\gamma$ if there exists a positive definite matrix $Q \in \mathscr{R}^{(n+m-1) \times(n+m-1)}$ satisfying (19). This implies that the consensus of system ((1), (2), (6)), (17) can be reached with $\left\|T_{z w}(s)\right\|_{\infty}<\gamma$ by protocol (6).

Remark 11. Theorem 10 gives the sufficient conditions of $H_{\infty}$ consensus for system ((1), (2), (6)), (17). In fact, the control parameters $k_{1}$ and $k_{2}$ also should satisfy condition (19). In practice, we can adjust the weights of adjacency matrix and the control parameters $k_{1}, k_{2}$ to make inequality (19) hold.

Remark 12. In the proof of Theorem 10, we first separate the agreement states from disagreement states by using a model transformation. Then, we studied the new states related to $H_{\infty}$ consensus problem instead of all states. This technique is very important for solving the problem of $H_{\infty}$ consensus.

\section{Simulations}

In this section, numerical simulations are given to illustrate the theoretical results obtained in the previous sections.

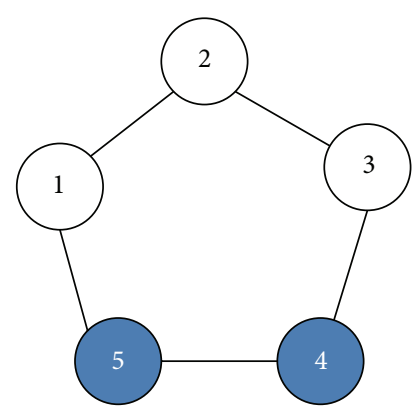

FIGURE 1: Network with five agents.

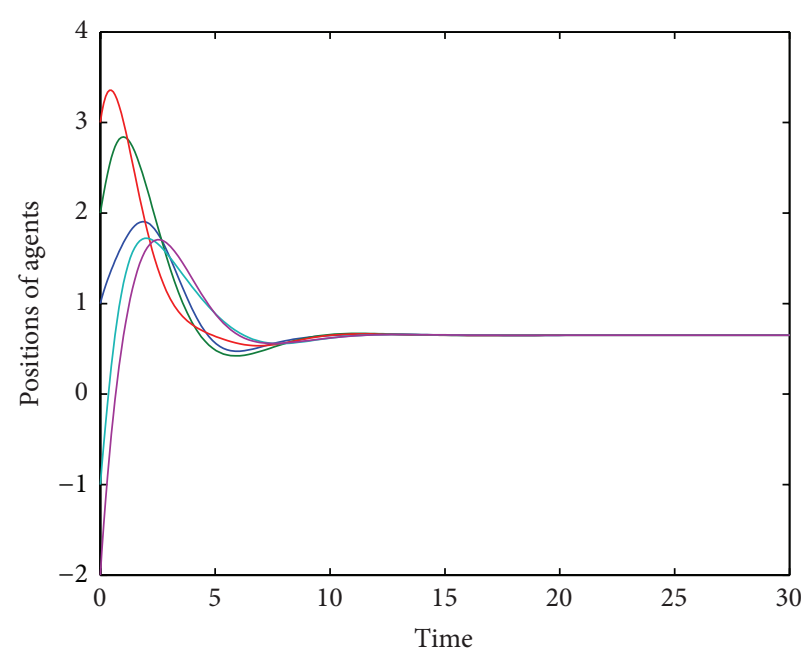

FIGURE 2: The positions of agents.

Figure 1 shows a graph with $n=5$ nodes. Suppose that the weight of each edge is 1 ; the vertices 1-3 denote the secondorder integrator agents and the vertices 4-5 denote the firstorder integrator agents. Assume $x(0)=[1,2,3-1,-2]^{T}$, $v(0)=[1,1.5,1.8]^{T}$, and $k_{1}=k_{2}=1$. Then Figures 2 and 3 show the positions of all agents and velocities of second-order integrator agents of system ((1), (2), (6)) with $w(t)=0$, respectively. And the consensus position is 0.6500 , which agrees well with the theoretical results obtained before. Choose the performance index $\gamma=0.53$. By MATLAB, it is easy to verify that linear matrix inequality (19) holds when $a_{12}=a_{23}=a_{34}=a_{45}=a_{51}=10.3$ and $Q=E$. Let

$$
\delta(t)= \begin{cases}1, & t<1 \\ 0, & t \geq 1\end{cases}
$$

Take $w_{i}(t)=0.8 \delta(t)$. Figure 4 shows the corresponding energy trajectories for the heterogeneous multiagent systems with external disturbance $w(t)$. That is, consensus is achieved with the performance $\left\|T_{z w}(s)\right\|_{\infty}<\gamma$. 


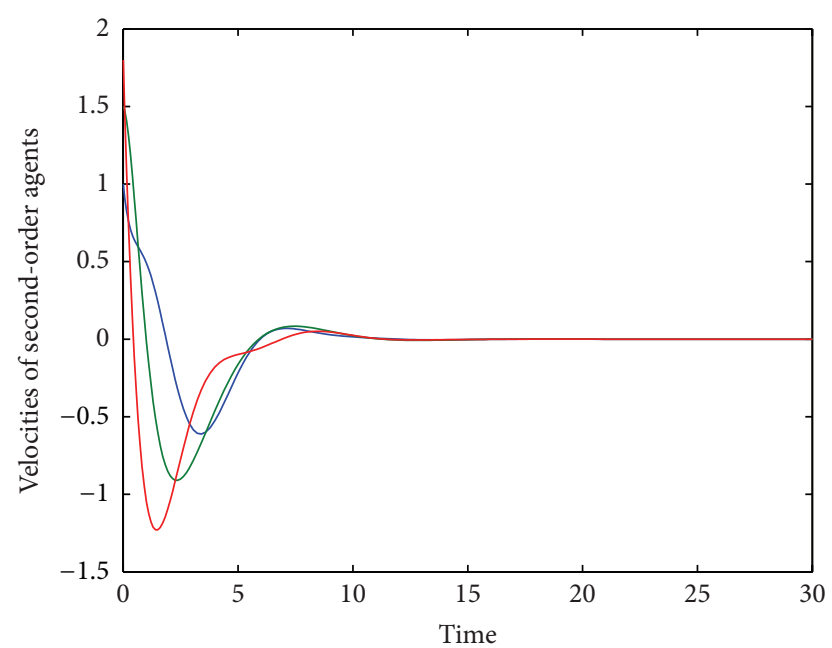

FIgURE 3: The velocities of second-order agents.

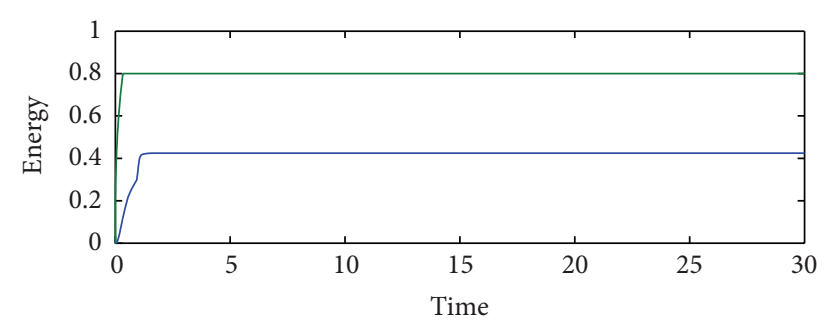

FIgURE 4: The energy trajectories of $z(t)$ and disturbance.

\section{Conclusions}

In this paper, we investigated the distributed coordination control problems of first- and second-order multiagent systems with external disturbances. A dynamic neighbor-based protocol is adopted for the agents. The consensus state is given by utilizing matrix theory and model transformation when external disturbance does not exist. Based on the consensus state, the control output is defined, and then sufficient conditions are derived to make all agents reach consensus while satisfying desired $H_{\infty}$ performance. Finally, simulation results are provided to demonstrate the effectiveness of the presented results. The method used in this paper can be easily extended to the heterogeneous multiagent systems with switching topologies. The future work will focus on the timedelay systems with directed network.

\section{Conflict of Interests}

The authors declare that there is no conflict of interests regarding the publication of this paper.

\section{Acknowledgments}

This work is supported by the National Natural Science Foundation (NNSF) of China (Grants nos. 61304155, 61203080, and 61573082), the Beijing Municipal Government Foundation for Talents (Grant no. 2012D005003000005), the Foundation of State Key Laboratory of Networking and Switching Technology (Grants nos. SKLNST2011105, SKLNST2013109), and State Key Laboratory of Intelligent Control and Decision of Complex Systems.

\section{References}

[1] W. Ren and Y. Cao, Distributed Coordination of Multi-Agent Networks: Emergent Problems, Springer, New York, NY, USA, 2010.

[2] T. Vicsek, A. Czirók, E. Ben-Jacob, I. Cohen, and O. Shochet, "Novel type of phase transition in a system of self-driven particles," Physical Review Letters, vol. 75, no. 6, pp. 1226-1229, 1995.

[3] A. Jadbabaie, J. Lin, and A. S. Morse, "Coordination of groups of mobile autonomous agents using nearest neighbor rules," IEEE Transactions on Automatic Control, vol. 48, no. 6, pp. 988-1001, 2003.

[4] Y. Hong, L. Gao, D. Cheng, and J. Hu, "Lyapunov-based approach to multiagent systems with switching jointly connected interconnection," IEEE Transactions on Automatic Control, vol. 52, no. 5, pp. 943-948, 2007.

[5] P. Lin and Y. Jia, "onsensus of a class of second-order multi-agent systems with time-delay and jointly-connected topologies," IEEE Transactions on Automatic Control, vol. 55, no. 3, pp. 778784, 2010.

[6] P. Lin and Y. Jia, "Multi-agent consensus with diverse timedelays and jointly-connected topologies," Automatica, vol. 47, no. 4, pp. 848-856, 2011.

[7] P. Lin and Y. Jia, "Consensus of second-order discrete-time multi-agent systems with nonuniform time-delays and dynamically changing topologies," Automatica, vol. 45, no. 9, pp. 21542158, 2009.

[8] P. Lin, W. Ren, and Y. Song, "Distributed multi-agent optimization subject to nonidentical constraints and communication delays," Automatica, In press.

[9] W. Zhang, J. Liu, D. Zeng, and T. Yang, "Consensus analysis of continuous-time second-order multi-agent systems with nonuniform time-delays and switching topologies," Asian Journal of Control, vol. 15, no. 5, pp. 1516-1523, 2013.

[10] Y.-P. Tian and C.-L. Liu, "Consensus of multi-agent systems with diverse input and communication delays," IEEE Transactions on Automatic Control, vol. 53, no. 9, pp. 2122-2128, 2008.

[11] G. Wen, Y. Yu, Z. Peng, and A. Rahmani, "Distributed finitetime consensus tracking for nonlinear multi-agent systems with a time-varying reference state," International Journal of Systems Science, 2014.

[12] J. Hu and W. X. Zheng, "Adaptive tracking control of leaderfollower systems with unknown dynamics and partial measurements," Automatica, vol. 50, no. 5, pp. 1416-1423, 2014.

[13] G. Ren, Y. Yu, and S. Zhang, "Leader-following consensus of fractional nonlinear multiagent systems," Mathematical Problems in Engineering, vol. 2015, Article ID 919757, 8 pages, 2015.

[14] P. Lin and Y. Jia, "Distributed rotating formation control of multi-agent systems," Systems and Control Letters, vol. 59, no. 10, pp. 587-595, 2010.

[15] P. Lin and W. Ren, "Constrained consensus in unbalanced networks with communication delays," IEEE Transactions on Automatic Control, vol. 59, no. 3, pp. 775-781, 2014. 
[16] P. Lin, K. Qin, Z. Li, and W. Ren, "Collective rotating motions of second-order multi-agent systems in three-dimensional space," Systems \& Control Letters, vol. 60, no. 6, pp. 365-372, 2011.

[17] Y. Zheng, Y. Zhu, and L. Wang, "Consensus of heterogeneous multi-agent systems," IET Control Theory \& Applications, vol. 5, no. 16, pp. 1881-1888, 2011.

[18] Y. Feng, S. Xu, F. L. Lewis, and B. Zhang, "Consensus of heterogeneous first- and second-order multi-agent systems with directed communication topologies," International Journal of Robust and Nonlinear Control, vol. 25, no. 3, pp. 362-375, 2015.

[19] Y. Liu, H. Min, S. Wang, Z. Liu, and S. Liao, "Distributed consensus of a class of networked heterogeneous multi-agent systems," Journal of the Franklin Institute. Engineering and Applied Mathematics, vol. 351, no. 3, pp. 1700-1716, 2014.

[20] L. Mo, Y. Niu, and T. Pan, "Consensus of heterogeneous multiagent systems with switching jointly-connected interconnection," Physica A, vol. 427, pp. 132-140, 2015.

[21] P. Lin, Y. Jia, and L. Li, "Distributed robust $H_{\infty}$ consensus control in directed networks of agents with time-delay," Systems \& Control Letters, vol. 57, no. 8, pp. 643-653, 2008.

[22] L. Mo and Y. Jia, " $H_{\infty}$ consensus of a class of high-order multi-agent systems with time- delay," IET Control Theory \& Applications, vol. 5, no. 1, pp. 247-253, 2011.

[23] L. Mo, Y. Zhou, and H. Zhou, " $L_{2}-H_{\infty}$ consensus of uncertain high-order multi-agent systems with a leader," Control Theory and Applications, vol. 29, no. 9, pp. 1125-1131, 2012.

[24] C. Godsil and G. Royle, Algebraic Graph Theory, Springer, New York, NY, USA, 2001.

[25] Y. Jia, Robust $\mathrm{H}_{\infty}$ Control, Science Press, Beijing, China, 2007 (Chinese). 


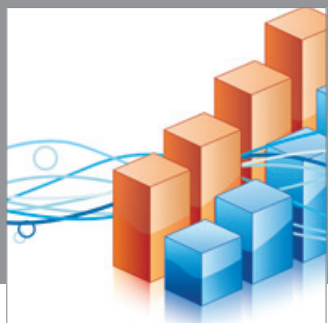

Advances in

Operations Research

mansans

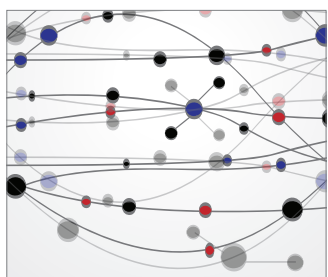

The Scientific World Journal
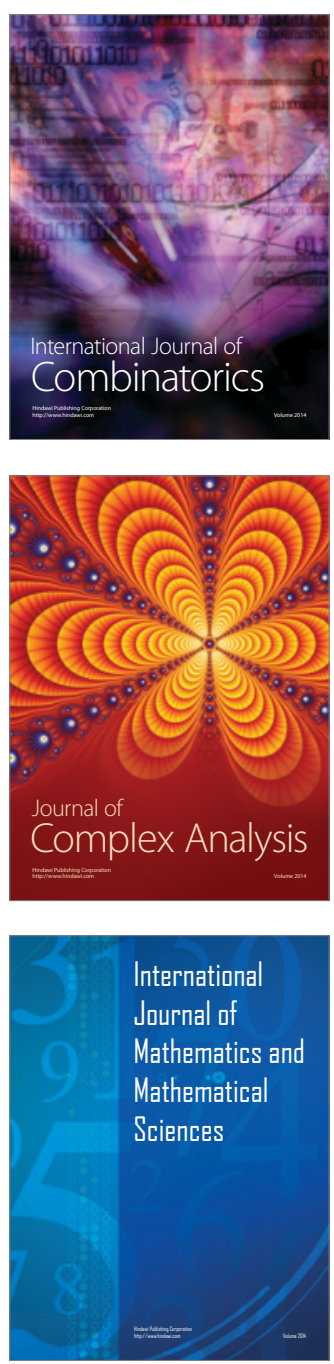
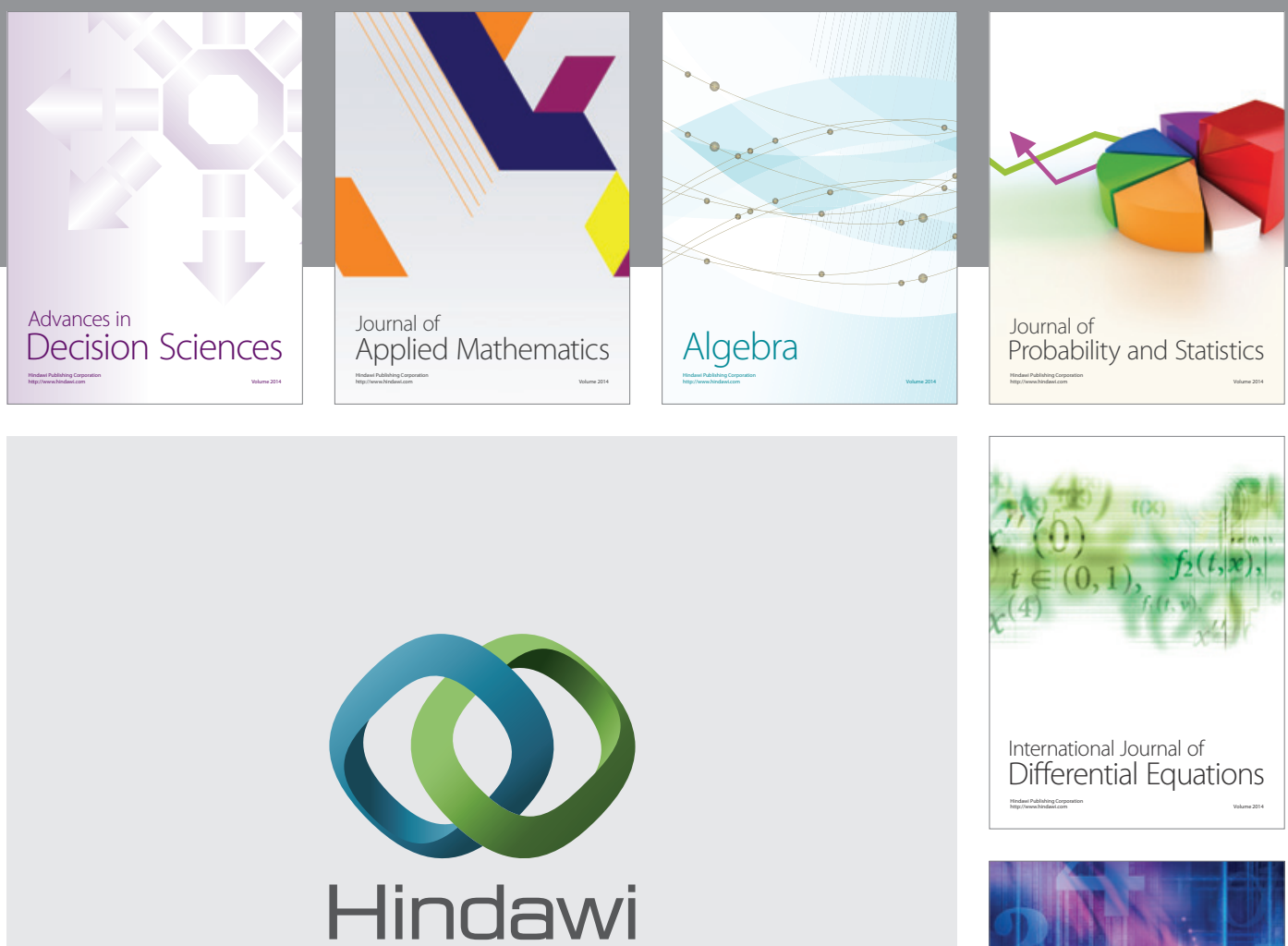

Submit your manuscripts at http://www.hindawi.com
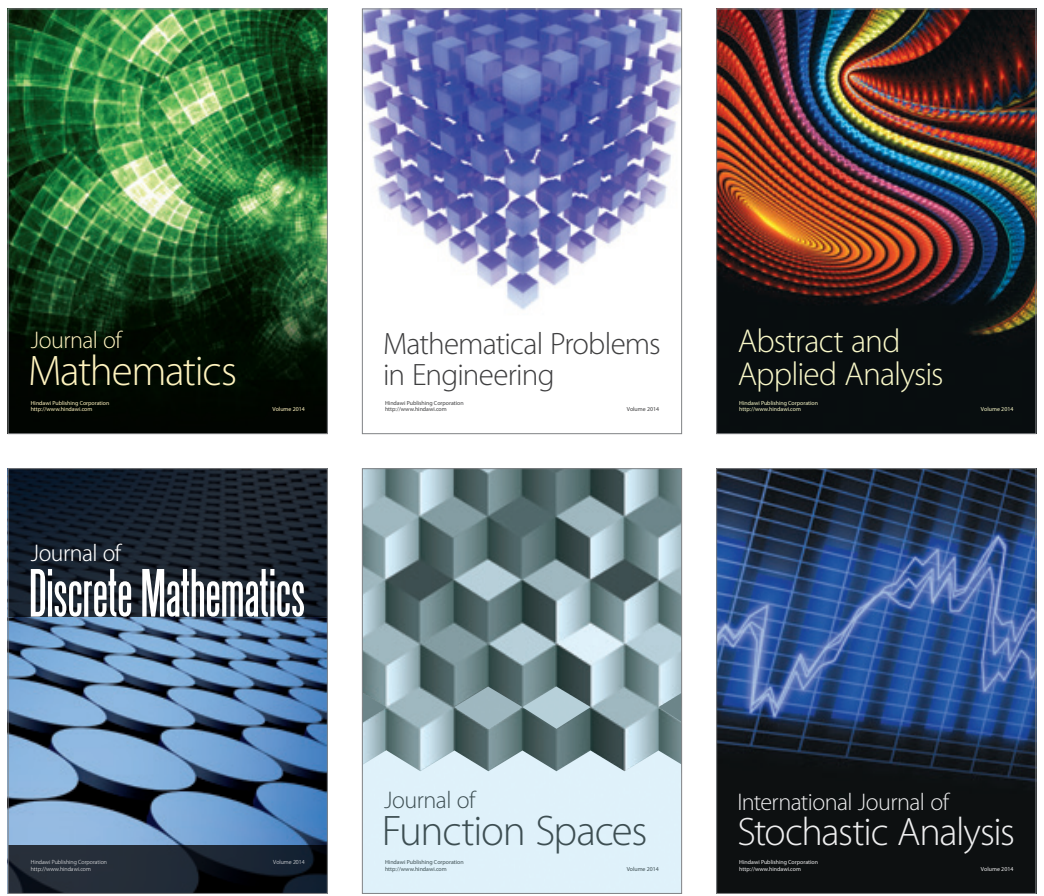

Journal of

Function Spaces

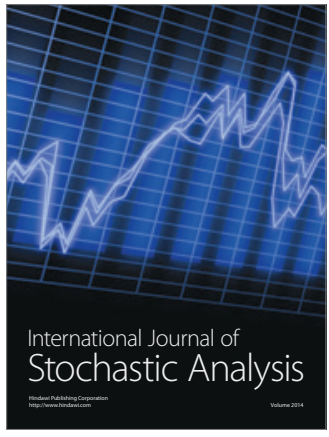

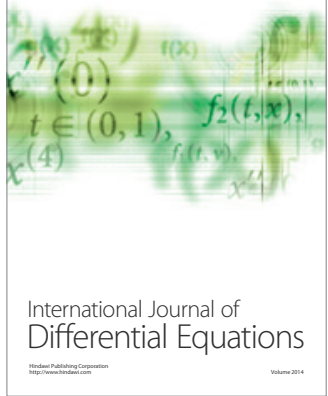
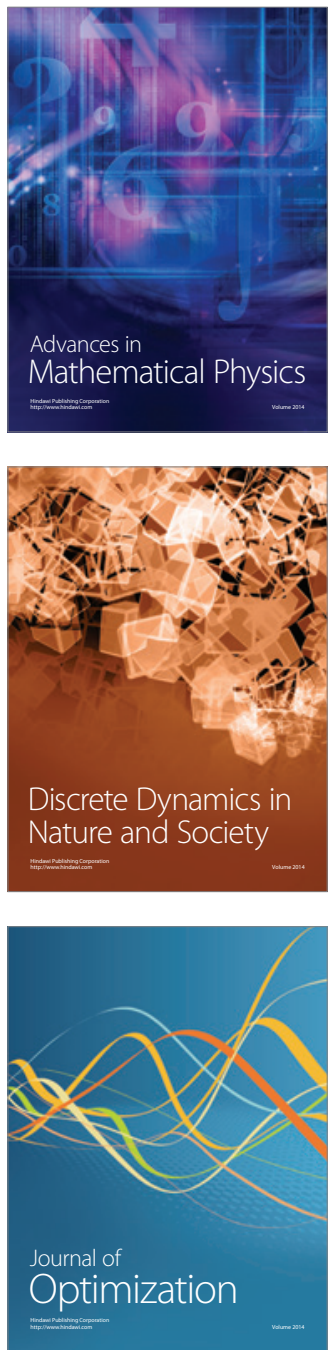